\title{
Accuracy of Noncontrast CT Brain in Detection of Cerebral Venous Sinus Thrombosis
}

\author{
Pipat Chiewvit ${ }^{*}$, Siri-on Tritrakarn1, Yongchai Nilanont ${ }^{2}$ \\ ${ }^{1}$ Department of Radiology, Faculty of Medicine Siriraj Hospital, Mahidol University, Bangkok, Thailand \\ ${ }^{2}$ Division of Neurology, Department of Medicine, Faculty of Medicine Siriraj Hospital, Mahidol University, Bangkok, Thailand \\ Email: *pipat8999@gmail.com
}

How to cite this paper: Chiewvit, P., Tritrakarn, S. and Nilanont, Y. (2021) Accuracy of Noncontrast CT Brain in Detection of Cerebral Venous Sinus Thrombosis. International Journal of Clinical Medicine, 12, 471-483.

https://doi.org/10.4236/ijcm.2021.1211043

Received: August 17, 2021

Accepted: November 20, 2021

Published: November 23, 2021

Copyright $\odot 2021$ by author(s) and Scientific Research Publishing Inc. This work is licensed under the Creative Commons Attribution International License (CC BY 4.0).

http://creativecommons.org/licenses/by/4.0/

\begin{abstract}
Background and Purpose: Increasing concern of cerebral venous thrombosis due to treatable and curable causes of stroke. The diagnosis of cerebral venous sinus thrombosis is challenged due to nonspecific clinical symptomatology. Patients may present at an emergency room with a variety of neurological conditions such as severe headache, weakness, seizure, etc. Neuroimaging, particularly noncontrast cranial computed tomography (NCCT), is an investigation of choice in differentiation and triage the patients for further treatment. CT is sensitive in the detection of acute thrombosis or blood clots in all regions of the body. We hypothesize that NCCT might be sensitive to diagnose cerebral venous thrombosis immediately. Materials and Methods: Retrospectively review the electronic database of our patients, there are 27 patients with cerebral sinus venous thrombosis (SVT) and 4 patients with cerebral deep venous thrombosis (DVT). Other 79 patients present with clinically diagnosed cerebral venous thrombosis but the final result can exclude cerebral venous thrombosis. We use MR imaging and CT venography as the gold standard. Independently reviewed by two neuroradiologists for CT direct sign and CT indirect signs that suggest SVT or DVT. CT direct signs for SVT and DVT are the presence of hyperdensity in the sinus venous or deep venous system (cord sign, attenuated vein sign) and CT indirect signs are the changes in brain parenchyma (brain edema, hemorrhagic infarction). Results: Sensitivity and specificity of NCCT in detection attenuated vein sign and diagnosis DVT are $75 \%$ and $100 \%$ whereas the sensitivity and specificity of NCCT in detection cord sign and diagnosis SVT are $43.8 \%$ and $99.7 \%$ as respectively. Conclusions: NCCT might not sensitive in detection of SVT without CT direct sign which needs further investigation. However, NCCT might beneficial for emergency conditions such as DVT patients, cortical vein thrombosis and also in SVT patients with the positive CT direct sign.
\end{abstract}




\section{Keywords}

Noncontrast CT, CT Brain, Cerebral Venous Sinus Thrombosis Cord Sign, Hyperattenuate Sign, CVT

\section{Introduction}

Cerebral venous thrombosis (CVT) is one of the causes of stroke even though less common comparable to stroke from arterial thrombosis. It still represents the common cause of stroke in young adults. Nowadays, CVT is increasingly encountered in daily practice.

Early diagnosis of cerebral venous thrombosis is important for the early treatment which can improve morbidity and mortality of the patients. Clinical symptoms of CVT are nonspecific [1] [2] with a wide range of severity such as headache, nausea, vomiting, dizziness up to neurological deficits and epilepsy [3] [4].

In contrast to the patients with stroke from arterial thrombosis, the symptoms of CVT patients are not present in the acute stage but slow progression [5] [6] [7]. Severe headache is the most common symptom which is counts as $74 \%$ 90\% of CVT patients and nonspecific [3] [8]. Early diagnosis of CVT should be in the lists of differential diagnoses whenever encountered in the young patients with unusual severe headache, with clinical arterial stroke and absence of risk factors, with intracranial hypertension or patients with hemorrhagic infarction on NCCT. The most common locations of intracranial dural venous sinuses are superior sagittal sinus followed by transverse sinus and sigmoid sinus as respectively [3] [4] [9].

Although CVT is not common, altogether with this condition awareness and new model of imaging CT scanner with rapid scan time, thin slices section, high resolution, the incidence of CVT is increase. Shorter time from onset of symptoms to diagnosis is with average of 7 days [3] [8].

Magnetic Resonance Imaging (MRI) and Magnetic Resonance venography (MRV) are the most sensitive imaging technique in diagnosis CVT [2] [8] [10]. The disadvantages of MRI are such as time consuming imaging technique, need patient cooperation particularly in the emergency condition [2] [6] [11]. So, multidetector computed tomography with venography (MDCTV) is of increased utility as the imaging modality in diagnosis CVT after inconclusive by NCCT.

NCCT can be performed faster and is suitable in the neuroemegency condition, still use as the first line of imaging modality in most centers. The radiologic patterns of NCCT in diagnosis of CVT (both SVT and DVT) are divided into direct signs and indirect signs. "Direct sign" refers to the visualization of the acute intraluminal blood clot or acute thrombosis that developed in the cranial venous system. If the thrombus formed within a week (acute stage), the attenuation value or CT density is higher than brain density and locates along course 
venous sinus, cortical veins or deep vein [5] [6]. After one to two weeks (subacute stage), the thrombus is getting decreased density to the same level of brain (isodensity) or lower than the brain (hypodensity) which might not be easily diagnosed by NCCT. "Indirect sign" refers to the brain parenchyma density changes resulting from cerebral venous thrombosis which could be brain edema or hemorrhagic infarction, etc. [6].

Previous study reports that the sensitivity and specificity of NCCT in detection direct sign in cerebral venous thrombosis for SVT is poor [7] which is contrary to our experiences in daily practice. The purpose of this study is to reevaluation the usefulness of NCCT in diagnosis CVT.

\section{Material and Method}

This study was approved by institutional review board committee and performed in Siriraj hospital.

\subsection{Definitions}

We defined the patients with cerebral venous thrombosis into two subgroups such as sinus venous thrombosis (SVT) which represents thrombosis in course dural sinus such as superior sagittal sinus, inferior sagittal sinus, transverse sinus, sigmoid sinus and deep venous thrombosis (DVT) which thrombosis is detected in those following veins as thalamostriate vein (TSV), internal cerebral veins (ICV), basal vein of Rosenthal (BVR), great vein of Galen (VG).

\subsection{Subjects}

Retrospectively review of the medical records of the patients who was diagnosed as cerebral venous thrombosis with undergone NCCT, CT venography (CTV) or MRV in past 3 years between 2009-2012.

Inclusion criteria: 1) NCCT must be performed; 2) Patients must have one of these following imaging studies such as MR Venography (MRV) either or multidetector CT venography (CTV). Four patients with DVT and 27 patients with SVT are included. Our control group is the 79 patients who had clinical cerebral venous thrombosis and underwent NCCT, however, eventually proven no cerebral venous thrombosis by either CTV or MRV. Similarly, the patients in control group must have inclusion criteria as present in Figure 1.

\subsection{Imaging}

All patients included in our study had undergone NCCT $(n=110)$. CT Venography were performed in 90 patients and MR venography were performed in 20 patients.

\subsection{Procedure Parameters}

All imaging procedures are undergone by the following machines and protocols:

All NCCT were undergone by one of the two 64-slices multidetector CT 


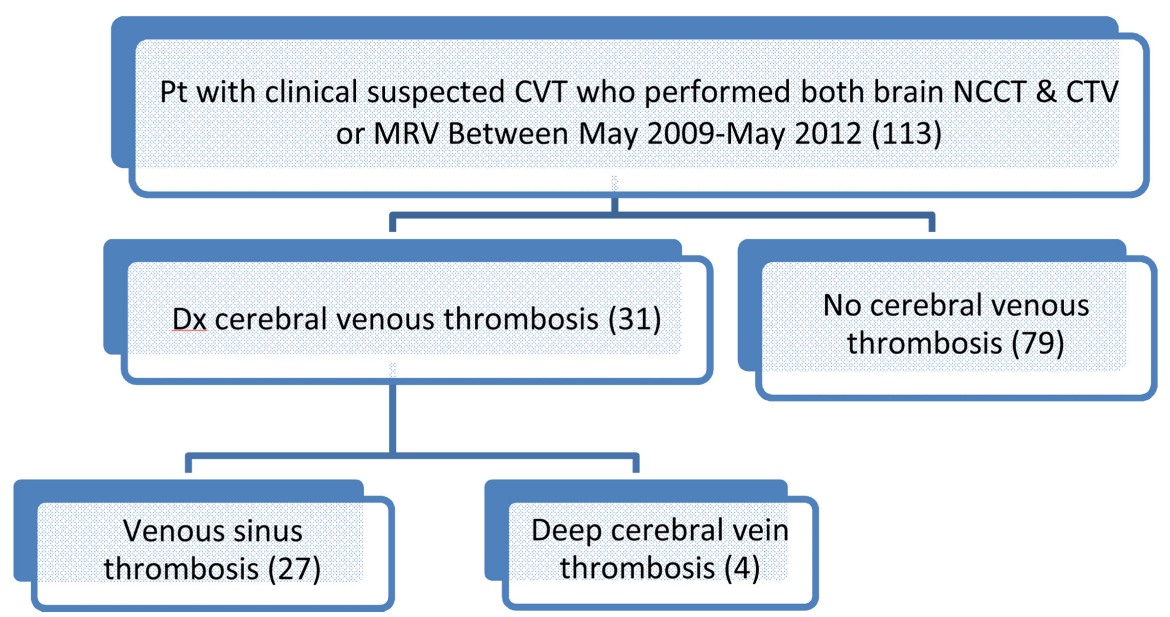

Figure 1. Flow diagram for patient inclusion in this study.

scanners, one from GE (GE heathcare, USA) and another from SIEMENS (Sensation 64; Siemens Medical Solutions, Erlangen, Germany). NCCT protocol used with $120 \mathrm{kV}, 300 \mathrm{mAs}$, collimation $2.5 \mathrm{~mm}$, slices thickness $1.25 \mathrm{~mm}$ and 5.0 $\mathrm{mm}$.

All CT Venography were undergone by the same 64-slices multidetector CT scanners. CTV protocol parameters as $120 \mathrm{kV}, 120$ - $140 \mathrm{mAs}$; collimation, 4 - 10 $\mathrm{mm}$., $80-100 \mathrm{~mL}$ contrast medium, injection rate $3-5 \mathrm{~mL} / \mathrm{s}$ and delay $35 \mathrm{~s}$.

All MR Venography were undergone by one of the two 3.0T MR scanner and 1.5 T MR scanner (Inter Achieva, Philips) with protocol parameters as follows:

T1-weighted SE-sequence (TR, $9.5 \mathrm{~ms}$; TE, $8 \mathrm{~ms}$; section thickness, $5 \mathrm{~mm}$; FOV, $230 \mathrm{~mm}$; matrix size, 256).

T2-weighted SE-sequence, (TR, $3000 \mathrm{~ms}$; TE, $90 \mathrm{~ms}$; section thickness, $5 \mathrm{~mm}$; FOV, $230 \mathrm{~mm}$; matrix size, 256).

DWI-sequence with b-values of 0 and 1000, and 3 gradient directions (TR, $2278 \mathrm{~ms}$; TE, $55 \mathrm{~ms}$; section thickness, $5 \mathrm{~mm}$; FOV, $230 \mathrm{~mm}$; matrix size, 128).

Fluid-attenuated inversion recovery (FLAIR) sequence (TR, 11,000 ms; TE, $120 \mathrm{~ms}$; section thickness, $5 \mathrm{~mm}$; FOV, $230 \mathrm{~mm}$; matrix size, 256).

Contrast enhanced MR venography (TR, $9.5 \mathrm{~ms}$; TE, $8 \mathrm{~ms}$; flip angle, $30^{\circ}$; section thickness, $5 \mathrm{~mm}$; FOV, $230 \mathrm{~mm}$; matrix size, 256).

\subsection{Image Review}

All of patients' imaging modalities study were interpreted by two neuroradiologists independently without available patients' clinical information and final diagnosis. Reading on PACS workstation in department of Radiology and recorded imaging informations in case record forms.

Recorded imaging data of the NCCT scan are the presence or absence of hyperdense sign (cord sign) in all sinus venous and cortical vein, hyperdense sign (attenuated vein sign) in deep vein. Presence or absence of brain edema, hemorrhagic infarct were also recorded. Then, the readers had to make conclusion for the diagnosis of SVT or DVT. After completing all NCCT interpreta- 
tions, the disconcordance cases are in consensus reading of the two readers with obtaining other imaging modalities such as CTV or MRV as well as clinical data of those cases.

\subsection{Statistical Analysis}

The statistical analysis of our recorded images data was performed by using of standard software (Excel and Access; Microsoft, STATA). The sensitivity and specificity of NCCT in the diagnosis of SVT and DVT as well as $95 \%$ confidence intervals were analyzed. Interobserver agreement of the hyperdense sign in sinuses or deep veins were analyzed with multirater values were calculated as described in the literature. The values can range from -1.0 to 1.0 , with -1.0 indicating perfect disagreement below chance, 0.0 indicating agreement equal to chance, and 1.0 indicating perfect agreement above chance.

\subsection{Results}

Four patients with DVT (all are women; age range from 34 to 46 years old; mean age, 43.7 years old). 27 patients with SVT (16 women, 11 men; age range 13 to 72 years old; mean age 42.2 years old. Most common of the clinical manifestations in our patients who were diagnosed as cerebral venous thrombosis were headache $(n=16)$, seizure $(n=8)$, paresis of variable degree $(n=7)$, alteration of consciousness $(n=2)$, aphasia $(n=2)$, paresthesia, diplopia, hemianopia, and behavioral change ( $n=1$ each) present in Table 1. Average time interval from earliest symptoms to diagnosis of cerebral venous thrombosis was 18.43 days.

No significant difference of the mean age of the patients with final diagnosis cerebral venous thrombosis and control group was 41.19 years and 35.09 years, ( $\mathrm{p}$ value $=0.122$ ) as respectively. No significant difference of the mean hematocrit level of each group was 32.31 and 30.97 , ( $\mathrm{p}$ value $=0.643$ ) as respectively present in Table 2.

Table 1. Clinical symptoms in patient with cerebral vein thrombosis.

\begin{tabular}{cc}
\hline Symptoms & Prevalence \\
\hline headache & $51.6 \%(n=16)$ \\
seizure & $25.8 \%(n=8)$ \\
paresis of variable degree & $22.5 \%(n=7)$ \\
alteration of consciousness & $6.4 \%(n=2)$ \\
aphasia & $6.4 \%(n=2)$ \\
paresthesia, diplopia, hemianopia, behavioral change & $(n=1$ each $)$ \\
\hline
\end{tabular}

Table 2. Mean age and hematocrit level of the included patients.

\begin{tabular}{cccc}
\hline & Control gr & CVT pos & P value \\
\hline Mean age & 35.09 & 41.19 & 0.122 \\
Mean HCt & 30.97 & 32.31 & 0.643 \\
\hline
\end{tabular}


90 out of 110 patients that have CTV as gold standard, 19 patients were sinovenous thrombosis, 3 patients were deep venous thrombosis and 68 patients were control group. Another 20 patients who have MRV as gold standard, 8 patients were sinovenous thrombosis, 1 patient was deep vein thrombosis and 11 patients were control group. Each reader had reviewed 220 imaging studies evaluation of total sinus venous and deep veins of 3300 venous structures. The result of interpretation in the patients with clinical cerebral venous thrombosis are presented in Tables 3-6.

Our study encountered the hyperattenuating sign in sinus venous structures and deep veins as follows: superior sagittal sinus (SSS) in 48\%, the right transverse sinuses (TS) $33.3 \%$, the left transverse sinuses (TS) $36.4 \%$, the right sigmoid sinuses in $16.7 \%$, left sigmoid sinus in $28.6 \%$, cortical vein $81.8 \%$, while the ISS showed no positive case, and the total deep veins thrombosis found in small

Table 3. Result from reading cerebral venous sinus and superficial cerebral vein by 2 readers.

\begin{tabular}{|c|c|c|c|c|c|c|c|c|}
\hline \multirow[b]{2}{*}{ Sinus } & \multirow{2}{*}{$\begin{array}{c}\text { Blinded } \\
\text { Cord } \\
\text { sign }\end{array}$} & \multicolumn{4}{|c|}{ Consensus reading } & \multirow{2}{*}{$\begin{array}{l}\text { Interobserver } \\
\text { agreement }\end{array}$} & \multirow{2}{*}{$\begin{array}{l}\text { Sensitivity } \\
(95 \% \mathrm{CI})\end{array}$} & \multirow{2}{*}{$\begin{array}{c}\text { Specitivity } \\
(95 \% \mathrm{CI})\end{array}$} \\
\hline & & tp & $\mathrm{fp}$ & tn & fn & & & \\
\hline SSS & 27 & 22 & 5 & 165 & 28 & 0.62 & 44 & 97 \\
\hline ISS & 0 & 0 & 0 & 220 & 0 & - & - & - \\
\hline LTS & 9 & 7 & 2 & 196 & 15 & 0.65 & 31.8 & 98.9 \\
\hline RTS & 12 & 7 & 5 & 181 & 17 & 0.47 & 29.1 & 97.3 \\
\hline LSS & 5 & 4 & 1 & 205 & 10 & 0.79 & 28.6 & 99.5 \\
\hline RSS & 4 & 2 & 2 & 206 & 10 & 0.49 & 16.7 & 99.0 \\
\hline cortical & 18 & 17 & 1 & 195 & 7 & 0.51 & 70.8 & 99.5 \\
\hline total & 75 & 59 & 16 & 1368 & 87 & 0.59 & 40.4 & 98.84 \\
\hline
\end{tabular}

Table 4. Result from reading deep cerebral veins by 2 readers.

\begin{tabular}{|c|c|c|c|c|c|c|c|c|}
\hline \multirow{2}{*}{$\begin{array}{l}\text { Deep } \\
\text { vein }\end{array}$} & \multirow{2}{*}{$\begin{array}{c}\text { Blinded } \\
\text { Dense } \\
\text { vein sign }\end{array}$} & \multicolumn{4}{|c|}{ Consensus reading } & \multirow{2}{*}{$\begin{array}{c}\text { Interobserver } \\
\text { agreement }\end{array}$} & \multirow{2}{*}{$\begin{array}{c}\text { Sensitivity } \\
(95 \% \mathrm{CI})\end{array}$} & \multirow{2}{*}{$\begin{array}{r}\text { Specitivity } \\
(95 \% \mathrm{CI})\end{array}$} \\
\hline & & tp & $\mathrm{fp}$ & tn & fn & & & \\
\hline SS & 2 & 2 & 0 & 214 & 4 & 1.00 & 33.3 & 100 \\
\hline LICV & 2 & 2 & 0 & 218 & 0 & 1.00 & 50 & 100 \\
\hline RICV & 2 & 2 & 0 & 218 & 0 & 1.00 & 50 & 100 \\
\hline VG & 2 & 2 & 0 & 218 & 0 & 1.00 & 100 & 100 \\
\hline LBVR & 0 & 0 & 0 & 220 & 0 & - & - & - \\
\hline RBVR & 0 & 0 & 0 & 220 & 0 & - & - & - \\
\hline LTSV & 2 & 2 & 0 & 218 & 0 & 0 & 50 & 100 \\
\hline RTSV & 2 & 2 & 0 & 218 & 0 & 0 & 50 & 100 \\
\hline total & 12 & 12 & 0 & 1744 & 4 & 1.0 & 75 & 100 \\
\hline
\end{tabular}


Table 5. Result of consensus reading cerebral venous sinus and superficial cerebral vein.

\begin{tabular}{|c|c|c|c|c|c|c|c|}
\hline \multirow{2}{*}{ Sinus } & \multirow{2}{*}{$\begin{array}{c}\text { Blinded } \\
\text { Cord sign }\end{array}$} & \multicolumn{4}{|c|}{ Consensus Reading } & \multirow{2}{*}{$\begin{array}{c}\text { Sensitivity } \\
(95 \% \mathrm{CI})\end{array}$} & \multirow{2}{*}{$\begin{array}{c}\text { Specificity } \\
(95 \% \mathrm{CI})\end{array}$} \\
\hline & & $\mathrm{TP}$ & FP & $\mathrm{TN}$ & FN & & \\
\hline SSS & 12 & 12 & 0 & 85 & 13 & $48(27.8-68.7)$ & $100(95.8-100)$ \\
\hline ISS & 0 & - & 0 & 110 & - & - & - \\
\hline LTS & 4 & 4 & 0 & 99 & 7 & $36.4(10.9-69.2)$ & $100(96.3-100)$ \\
\hline RTS & 4 & 4 & 0 & 98 & 8 & $33.3(9.9-65.1)$ & $100(96.3-100)$ \\
\hline LSS & 2 & 2 & 0 & 103 & 5 & $28.6(3.6-71)$ & $100(96.3-100)$ \\
\hline RSS & 1 & 1 & 0 & 104 & 5 & $16.7(0.4-64.1)$ & $100(96.5-100)$ \\
\hline Cortical & 9 & 9 & 1 & 97 & 3 & $81.8(42.8-94.5)$ & $98.9(96.3-100)$ \\
\hline Total & 32 & 32 & 2 & 695 & 41 & 43.8 & $99.7(95.9-100)$ \\
\hline
\end{tabular}

Table 6. Result of consensus reading deep cerebral vein.

\begin{tabular}{|c|c|c|c|c|c|c|c|}
\hline \multirow{2}{*}{ Deep vein } & \multirow{2}{*}{$\begin{array}{c}\text { Blinded } \\
\begin{array}{c}\text { Dense vein } \\
\text { sign }\end{array}\end{array}$} & \multicolumn{4}{|c|}{ Consensus reading } & \multirow{2}{*}{$\begin{array}{c}\text { Sensitivity } \\
(95 \% \mathrm{CI})\end{array}$} & \multirow{2}{*}{$\begin{array}{c}\text { Specitivity } \\
(95 \% \mathrm{CI})\end{array}$} \\
\hline & & $\mathrm{tp}$ & $\mathrm{fp}$ & tn & fn & & \\
\hline SS & 1 & 1 & 0 & 107 & 2 & 50 & 100 \\
\hline LICV & 1 & 1 & 0 & 109 & 0 & 100 & 100 \\
\hline RICV & 1 & 1 & 0 & 109 & 0 & 100 & 100 \\
\hline VG & 1 & 1 & 0 & 109 & 0 & 100 & 100 \\
\hline LBVR & 0 & 0 & 0 & 110 & 0 & - & - \\
\hline RBVR & 0 & 0 & 0 & 110 & 0 & - & - \\
\hline LTSV & 1 & 1 & 0 & 109 & 0 & 100 & 100 \\
\hline RTSV & 1 & 1 & 0 & 109 & 0 & 100 & 100 \\
\hline total & 6 & 6 & 0 & 872 & 2 & $75(47.8-95.7)$ & $100(96.7-100)$ \\
\hline
\end{tabular}

number, albeit the sensitivity for overall DVT was about $75 \%$ (Table 5 and Table 6) (Figure 2). After the consensus reading, there were 2 false-negative diagnoses of DVT, 41 false-negative diagnosed of SVT, and 1 false-positive diagnoses in the SVT group. Therefore, the sensitivity of NCCT in detection hyperattenuating vein sign for the deep vein thrombosis were $50 \%$ and the specificity was $100 \%$ whereas the sensitivity and specificity of NCCT in detection the hyperdense sign (cord sign) for the diagnosis of SVT were $43.8 \%$ and $99.7 \%$ respectively.

Summary of result in interpretation sinus venous thrombosis and deep vein thrombosis as well as false positive and false negative cases are presented in $\mathrm{Ta}$ ble 3 and Table 4 . The sensitivity and specificity of attenuated vein sign in patients with DVT were $75 \%$ and $100 \%$ whereas the sensitivity and specificity of cord sign in patients with SVT were $43.8 \%$ and $99.7 \%$ as respectively. The interobserver agreement of attenuated vein sign was 0.59 (range $0.47-0.79$; Table 3 ) 


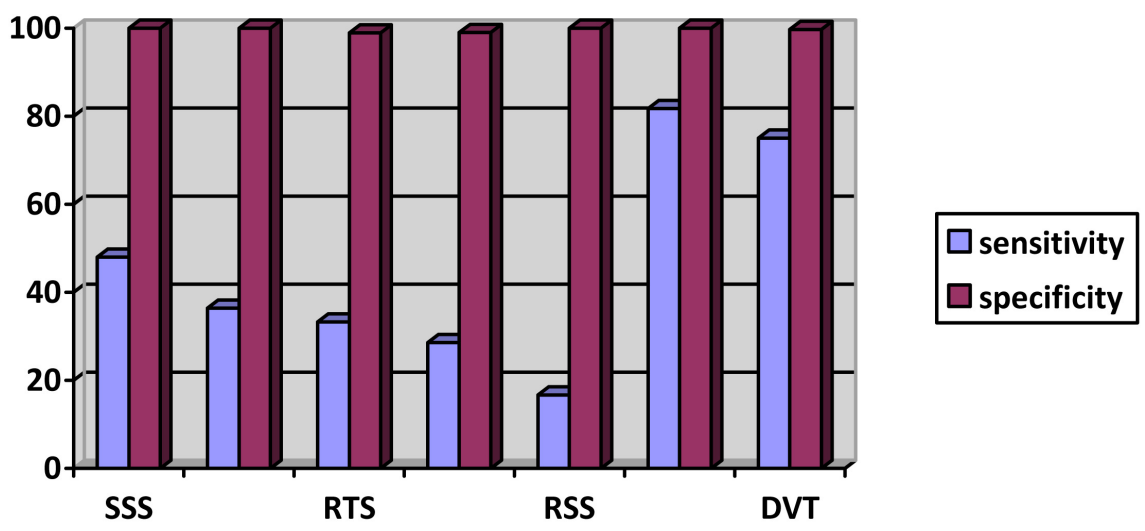

Figure 2. Summary sensitivity and specificity of NCCT brain in diagnosis cerebral venous thrombosis (SSS = superior sagittal sinus, LTS = left transverse sinus, RTS = right transverse sinus, LSS = left sigmoid sinus, RSS = right sigmoid sinus, CV = cortical veins, DVT = deep cerebral vein thrombosis).

and 1.0 for the cord sign (Table 4). NCCT detected intracerebral edema was found in noncontrol group 13 cases (41.9\%), infarction 8 cases (25.8\%), and hemorrhage or hemorrhagic transformations in 8 cases (25.8\%).

\section{Discussion}

Early treatments for the patients with CVT have been proved to obtain an excellent clinical results. Thus, early diagnosis is one of the key success factor of the whole process of patients care particularly in the patients with deep cerebral veins thrombosis [12] [13]. Until the present day, there is long duration time from onset of symptoms to diagnosis of CVT. The main reason is the great variability of the clinical manifestation of CVT patients [14] [15]. Sending D-dimer might not be helpful in diagnosis CVT due to high negative predictive value, normal study could not exclusion of the CVT patients, further indicate the importance of imaging [3]. Catherized cerebral angiography had been the gold standard imaging for diagnosis and follow up treatment in previous days. Nowadays, the procedure of direct cerebral angiography may be considered as invasive imaging procedure. Improvement of technology of noninvasive imaging such as MRI and CT imaging have led to good quality in the visualization of vascular structures either arterial or venous sides with high diagnostic value [2] [3] [10] [16] [17]. However, daily clinical practice in most centers, noncontrast CT brain scan is the forefront imaging study for the patients who have nonspecific neurological symptoms particularly in the emergency departments. The reasons for popularity in usage NCCT study are due to short scan time study, no need for patient preparation nor fully cooperation of the patients nor sedative drugs prescription. No risk of intravenously contrast medium reaction, neprotoxitcity. NCCT still provides good resolution image quality for rapidly diagnosis and prompt further treatment. The patients with CVT are in the same conditions. To review NCCT imaging of our patients in all CVT groups and control groups, the two readers interpreted imaging without clinical information of ce- 
rebral venous thrombosis. We found that hyperdensity in the cortical veins is the direct CT evidence of detection intraluminal thrombosis in the cortical veins that called "hyperattenuating vein sign" that similar in deep veins system (Figure 3 and Figure 4) is more sensitive CT sign than the detection of thrombus in the dural venous sinus that called "cord sign" (Figure 5). The sensitivity of hyperattenuated vein sign for cortical veins and deep vein thrombosis are $70.8 \%$ and $75 \%$ whereas cord sign is only $43.8 \%$. Even though, the sensitivity of cord sign by NCCT in diagnosis sinus venous thrombosis is too low in the present study, however, is similar to previous studies sensitivity $25 \%$ - 56\% [10] [15]. We suggest that the negative case by NCCT in the patients suspected cerebral venous thrombosis might not exclude sinus venous thrombosis (SVT). One main reason of low sensitivity NCCT in SVT found in our study is due to intrasinus thrombosis clot density, if it was isodensity to the brain, it is not possible to diagnosis by means of NCCT (Figure 6 and Figure 7). The density of thrombus is directly related to age of thrombus, the isodensity thrombus is not in acute stage but to subacute or chronic stage which might reflect to variety of clinical symptoms and nonspecific, delayed imaging can occur. Our findings are different from previous study by Alsafi A. et al. [18] that those cases are in acute stage hyperattenuated ( $\mathrm{HU}>67)$ greater than density of the brain, we found some of cases that isodensity blood clot in the sinuses (Figure 6 and Figure 7) which can cause false negative in the study. Limitations of the present study are first, some cases have only thick slice of NCCT (12 cases), which could cause an increasing rate of false negative results, second, nonhomogenously use standard reference imaging either CTV or MRV and third, number of patient

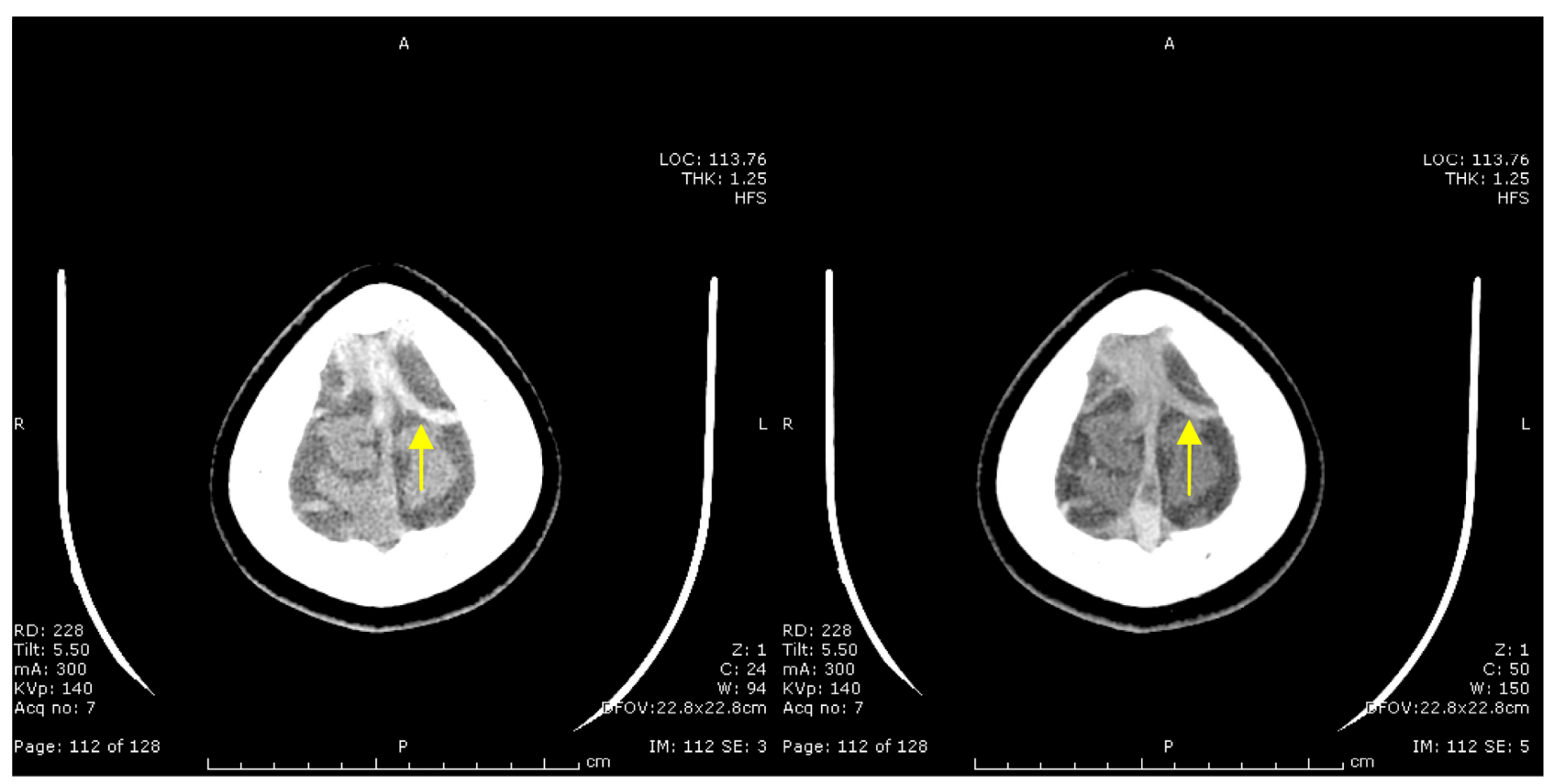

Figure 3. A 14-year-old male patient, systemic lupus erythematosus (SLE) with thrombosis of superior sagittal sinus (SSS) and cortical vein, who presented with headache for 6 months. The NCCT scan shows a true-positive cord sign in the SSS and cortical vein (arrows). 


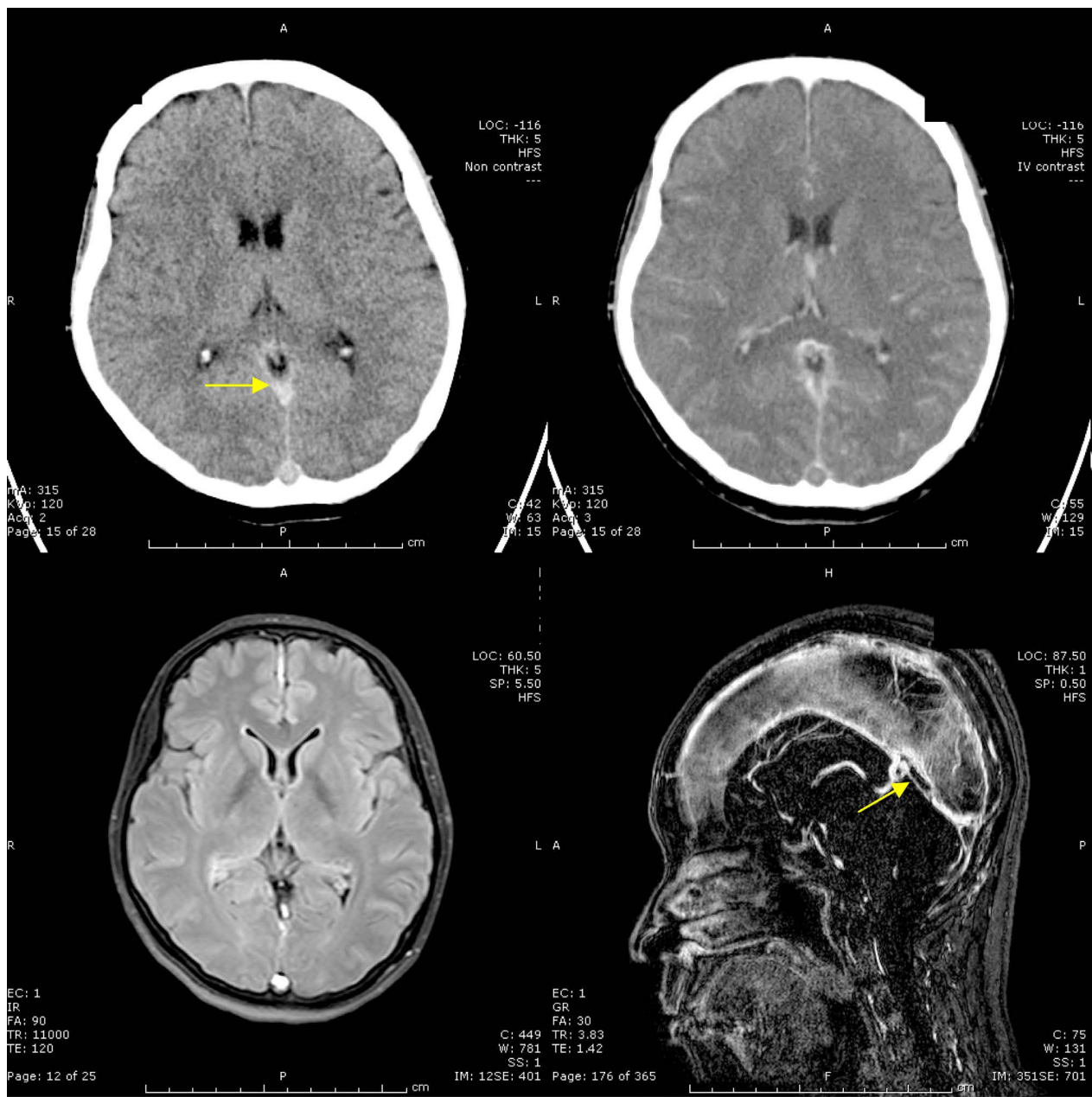

Figure 4. A 44-year-old female patient with venous thrombosis of the SSS, SS, VG. The NCCT scan demonstrates an attenuated vein sign in SS, VG (arrows). The thrombosis is visualized indirectly by demonstration of filling defects in the SS, VG (arrow) on the CTV and MRV.

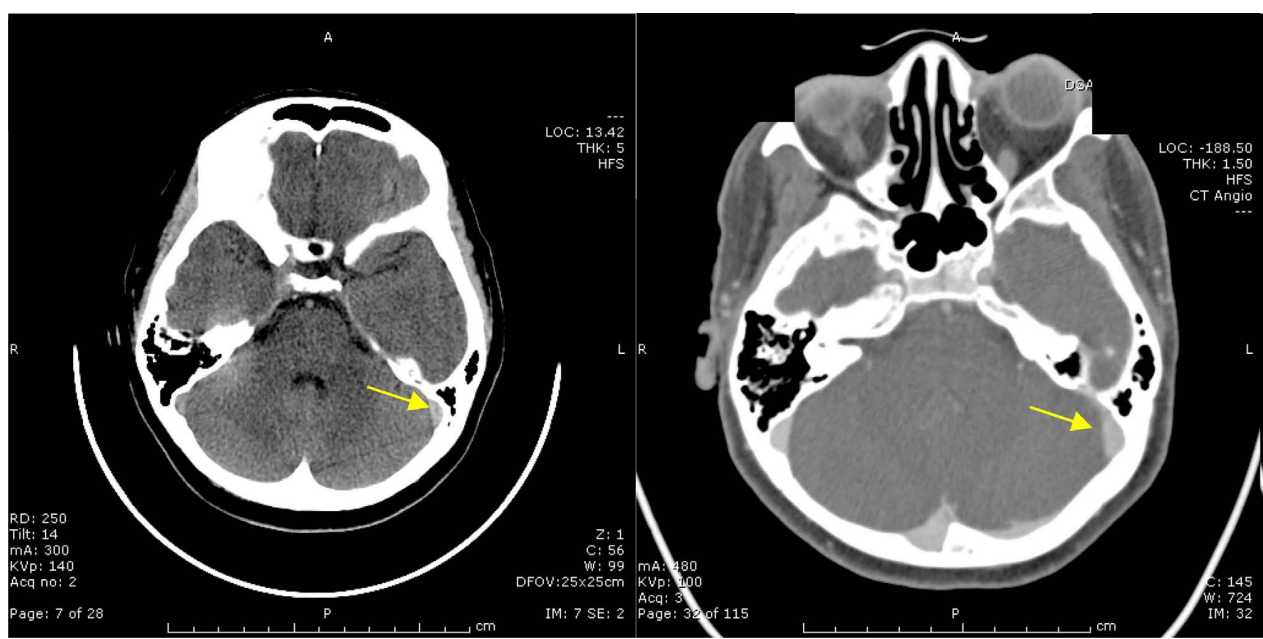

Figure 5. A 31-year-old female patient in the control group, with sepsis. There are suspected hyperattenuated lesion at SSS and both transverse sinus on NCCT (arrows). This finding was interpreted as a cord sign by all readers, resulting in the false-positive diagnosis of a SVT in this patient. Her Hct was 36.7. 


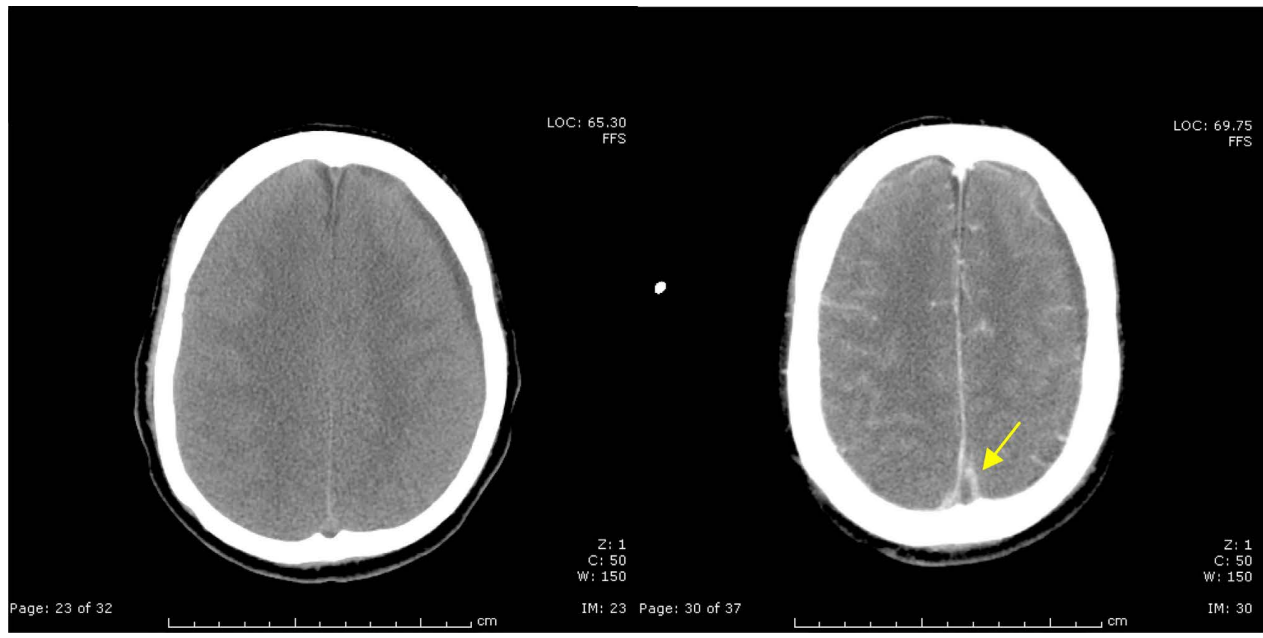

Figure 6. A 39-year old female patient presented with a progressive headache 3 weeks and status epilepticus. The NCCT scan shows no hyperattenuated sign (false-negative). The CTV scan demonstrates contrast-filling defects in posterior part of SSS (arrow), indicating CVT. There is also a chronic subdural collection at left cerebral hemisphere.

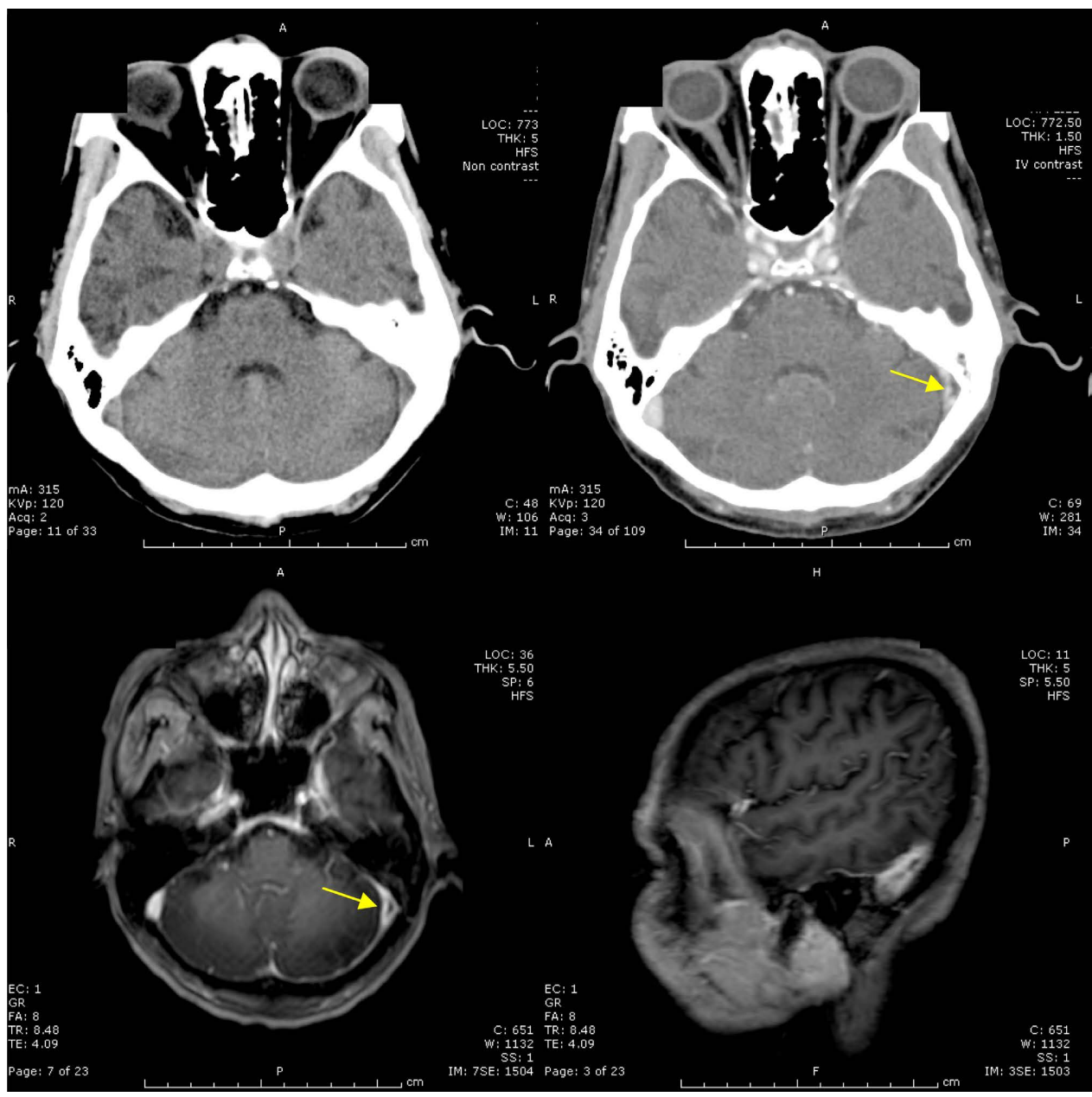

Figure 7. A 47-year old male patient presented with a progressive headache. The NCCT scan shows no hyperattenuated sign (false-negative). MRV demonstrates contrast-filling defects in Left transverse sinus (arrow), indicating CVT. 
population is relatively small.

\section{Conclusion}

NCCT has an impact on the diagnosis of deep cerebral vein thrombosis (DVT) as well as cortical vein thrombosis with high sensitivity and specificity. Absence of hyperattenuating vein signs and parenchymatous changes are less suspicious of deep cerebral vein thrombosis. On contrary, NCCT has poor sensitivity in detection of sinus venous thrombosis (SVT), negative detection suggests to be further investigated by CTV or MRV.

\section{Conflicts of Interest}

The authors declare no conflicts of interest regarding the publication of this paper.

\section{References}

[1] Karthikeyan, D., Vijay, S., Kumar, T., et al. (2004) Cerebral Venous ThrombosisSpectrum of CT Findings. Neuroradiology, 14, 129-137.

[2] Chiewvit, P., Piyapittayanan, S. and Poungvarin, N. (2011) Cerebral Venous Thrombosis: Diagnostic Dilemma. Neurology International, 3, e13. https://doi.org/10.4081/ni.2011.e13

[3] Ferro, J.M., Canhao, P., Stam, J., et al. (2004) Prognosis of Cerebral Vein and Dural sinus Thrombosis: Results of the International Study on Cerebral Vein and Dural sinus Thrombosis (ISCVT). Stroke, 35, 664-670. https://doi.org/10.1161/01.STR.0000117571.76197.26

[4] Raam, R. and Tabatabai, R.R. (2021) Headache in the Emergency Department: Avoiding Misdiagnosis of Dangerous Secondary Causes, an Update. Emergency Medicine Clinics of North America, 39, 67-85. https://doi.org/10.1016/j.emc.2020.09.004

[5] Lee, S.K., Kim, B.S. and TerBrugge, K.G. (2002) Clinical Presentation, Imaging and Treatment of Cerebral Venous Thrombosis (CVT). Interventional Neuroradiology, 8, 5-14. https://doi.org/10.1177/159101990200800102

[6] Saposnik, G., Barinagarrementeria, F., Brown, R.D. and Bushnell, C.D. (2011) Diagnosis and Management of Cerebral Venous Thrombosis. Stroke, 42, 1158-1192. https://doi.org/10.1161/STR.0b013e31820a8364

[7] Idiculla, P.S., Gurula, D., Palanisamy, M., Vijayakumar, R., Dhandapani, S. and Nagarajan, E. (2020) Cerebral Venous Thrombosis: A Comprehensive Review. European Neurology, 83, 369-379. https://doi.org/10.1159/000509802

[8] Stam, J. (2005) Thrombosis of the Cerebral Veins and Sinuses. The New England Journal of Medicine, 352, 1791-1798. https://doi.org/10.1056/NEJMra042354

[9] Rischall, M.A., Boegel, K.H., Palmer, C.S., Knoll, B. and McKinney, A.M. (2016) MDCT Venographic Patterns of Dural Venous Sinus Compromise after Acute Skull Fracture. American Journal of Roentgenology, 207, 852-858. https://doi.org/10.2214/AJR.15.15972

[10] Van Dam, L.F., Van Walderveen, M.A.A., Kroft, L.J.M., Kruyt, N.D. and Wermer, M.J.H. (2020) Current Imaging Modalities for Diagnostic Cerebral Vein Thrombosis-A Critical Review. Thrombosis Research, 189, 132-139. 
https://doi.org/10.1016/j.thromres.2020.03.011

[11] Leach, J.L., Fortuna, R.B., Jones, B.V. and Gaskill-Shipley, M.F. (2006) Imaging of Cerebral Venous Thrombosis: Current Techniques, Spectrum of Findings, and Diagnostic Pitfalls. Radiographic, 26, S19-S43. https://doi.org/10.1148/rg.26si055174

[12] Qu, H. and Yang, M. (2013) Early Imaging Characteristics of 62 Cases of Cerebral Venous Sinus Thrombosis. Experimental and therapeutic Medicine, 5, 233-236. https://doi.org/10.3892/etm.2012.796

[13] Caneodo-Antelo, M., Baleato-Gonzalez, S., Mosqueira, A.J., Casas-Martineze, J., et al. (2019) Radiologic Clues to Cerebral Venous Thrombosis. Radiographics, 39, 161-1628. https://doi.org/10.1148/rg.2019190015

[14] Luo, Y., Tian, X. and Wang, X. (2018) Diagnosis and Treatment of Cerebral Venous Thrombosis: A Review. Frontiers in Aging Neuroscience, 10, Article No. 2. https://doi.org/10.3389/fnagi.2018.00002

[15] Lee, E.J.Y. (2002) The Empty Delta Sign. Radiology, 224, 788-789. https://doi.org/10.1148/radiol.2243990978

[16] Randolph, J.J. (2005) Free-Marginal Multirater Kappa: An Alternative to Fleiss' Fixedmarginal Multirater Kappa. Joensuu University Learning and Instruction Symposium, Joensuu, Finland, 14-15 October 2005, 1-20.

[17] Gaikwad, A.B., Mudalgi, B.A., Patankar, K.B., Patil, J.K. and Ghongade, D.V. (2008) Diagnostic Role of 64-Slice Multidetector Row CT Scan and CT Venogram in Cases of Cerebral Venous Thrombosis. Emergency Radiology, 15, 325-333. https://doi.org/10.1007/s10140-008-0723-4

[18] Alsafi, A., Lakhami, A., Jones, I.C. and Lobotesis, K. (2015) Cerebral Venous Sinus Thrombosis, a Nonenhanced CT Diagnosis? Radiology Research and Practice, 2015, Article ID: 581437. https://doi.org/10.1155/2015/581437 